PROCEEDINGS OF THE

AMERICAN MATHEMATICAL SOCIETY

Volume 137, Number 4, April 2009, Pages 1239-1243

S 0002-9939(08)09712-8

Article electronically published on October 9, 2008

\title{
ON GENUS CHANGE IN ALGEBRAIC CURVES OVER IMPERFECT FIELDS
}

\author{
STEFAN SCHRÖER \\ (Communicated by Ted Chinburg)
}

\begin{abstract}
We give a new proof, in scheme-theoretic language, of Tate's classical result on genus change of curves over imperfect fields in characteristic $p>0$. Namely, for normal geometrically integral curves, the difference between arithmetic and geometric genus over the algebraic closure is divisible by $(p-1) / 2$.
\end{abstract}

\section{INTRODUCTION}

A peculiar feature of geometry in characteristic $p>0$ is that a regular scheme $X$ of finite type over an imperfect field $K$ may cease to be regular after a purely inseparable base change. This striking behavior easily appears for the generic fiber of morphisms $f: S \rightarrow B$ between smooth schemes over algebraically closed ground fields: Here $K=\kappa(B)$ is the function field of $B$, and $X=S_{K}$ is the generic fiber in the sense of scheme theory. When it comes to the classification of fibrations, for example in the Enriques classification of surfaces, the theory of Albanese maps, or the minimal model program, it is crucial to understand this behavior.

The simplest situation is that $X$ is a proper normal curve over an imperfect field $K$. If $K \subset K^{\prime}$ is a purely inseparable field extension, the induced curve $X^{\prime}=X \otimes_{K} K^{\prime}$ is not necessarily normal. Let $\tilde{X}^{\prime}$ be its normalization. Then the genus $\tilde{g}=h^{1}\left(\mathcal{O}_{\tilde{X}^{\prime}}\right)$ may be strictly smaller than the genus $g=h^{1}\left(\mathcal{O}_{X}\right)$ of our original curve. Tate [8] proved that such a genus change is not arbitrary.

Theorem (Tate). The difference $g-\tilde{g}$ is divisible by $(p-1) / 2$.

In particular, this puts an upper bound on the characteristic in terms of the possible genera occurring in genus change situations. This is a prominent manifestation of the intuitive principle that a given geometrical deviation in positive characteristics in a fixed dimension should occur only at finitely many primes. Example: Quasielliptic fibrations (the case $g=1, \tilde{g}=0$ ) are possible only at prime $p=2$ and $p=3$.

In 1952, Tate naturally stated and proved his result in the language of function fields and repartitions. In my opinion, it is desirable to have a proof in the modern language of schemes as well. Indeed, in the special case $\tilde{g}=0$, Shepherd-Barron 7 found such a proof for the inequality $g \geq(p-1) / 2$, using vector bundles on algebraic surfaces. The goal of this paper is to give an easy direct proof of Tate's

Received by the editors April 11, 2007, and, in revised form, April 17, 2008.

2000 Mathematics Subject Classification. Primary 14H20.

(C)2008 American Mathematical Society

Reverts to public domain 28 years from publication 
result, using only relative dualizing sheaves and relative Frobenius maps for curves. The result takes the following form:

Theorem. Let $Y$ be the normalization of the Frobenius pullback $X^{(p)}$. Then the degree of the relative dualizing sheaf $\omega_{Y / X^{(p)}}$ is divisible by $p-1$.

Our proof hinges on a result of Kiehl and Kunz [6], which implies that a finite universal homeomorphism between regular curves locally admits $p$-bases. We expect that this approach, with appropriate Gorenstein assumptions, will be useful in higher dimensions as well.

\section{Normalization after Frobenius pullback}

Let $K$ be a field of characteristic $p>0$, and let $X$ be a normal $K$-scheme of finite type. Throughout, we assume that $X$ is geometrically integral; that is, the induced schemes $X^{\prime}=X \otimes_{K} K^{\prime}$ remain integral for all base field extensions $K \subset K^{\prime}$. The scheme $X^{\prime}$, however, is not necessarily normal. In this section, we shall collect some useful facts about the normalization of $X^{\prime}$. Our first observation is:

Lemma 1.1. Let $K \subset K^{\prime}$ be a base field extension. Set $X^{\prime}=X \otimes_{K} K^{\prime}$, and let $Y^{\prime} \rightarrow X^{\prime}$ be the normalization. Then the $K^{\prime}$-scheme $Y^{\prime}$ is geometrically integral.

Proof. Geometric irreducibility and geometric reducedness easily follow from [3, Proposition 4.5.9 and Proposition 4.6.1, respectively].

Next, we consider the Frobenius pullback $X^{(p)}$, which is defined by the Cartesian square

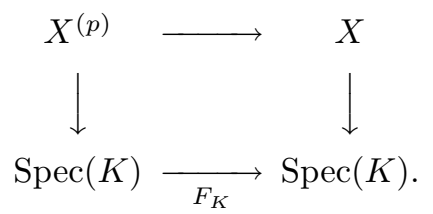

Here $F_{K}$ denotes the absolute Frobenius morphism, which corresponds to the Frobenius map $\operatorname{Fr}: K \rightarrow K, \lambda \mapsto \lambda^{p}$. The $K$-scheme $X^{(p)}$ is of finite type and geometrically integral, but not necessarily normal. In any case, the Frobenius pullback is closely related to the original normal scheme $X$ via the relative Frobenius morphisms $F_{X / K}: X \rightarrow X^{(p)}$. This is a finite universal homeomorphism, coming from the commutative square

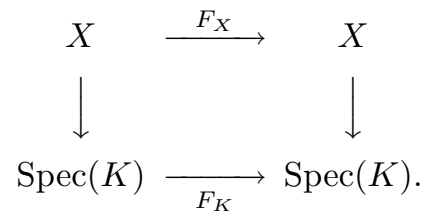

Using iterated Frobenius maps, we obtain similarly the iterated Frobenius pullback $X^{\left(p^{n}\right)}$, together with the iterated relative Frobenius morphism $F_{X / K}^{n}: X \rightarrow X^{\left(p^{n}\right)}$. The following observation will be useful:

Lemma 1.2. There is an integer $n_{0} \geq 0$ such that for all integers $n \geq n_{0}$, the normalization of $X^{\left(p^{n}\right)}$ is geometrically normal. 
Proof. Clearly, it suffices to find one integer $n \geq 0$ so that the normalization of $X^{\left(p^{n}\right)}$ is geometrically normal. To do so, choose a perfect closure $K \subset K^{p^{-\infty}}$. Set $Z=X \otimes_{K} K^{p^{-\infty}}$, and let $\nu: \tilde{Z} \rightarrow Z$ be the normalization. The scheme $\tilde{Z}$ is geometrically normal over $K^{p^{-\infty}}$, because the latter is perfect. According to 4, Theorem 8.8.2], there is an intermediate field $K \subset K^{\prime} \subset K^{p^{-\infty}}$ that is finite over $K$, so that the scheme $\tilde{Z}$ and the morphism $\nu: \tilde{Z} \rightarrow Z$ over $K^{p^{-\infty}}$ are induced from a scheme $\tilde{X}^{\prime}$ and a morphism $\nu^{\prime}: \tilde{X}^{\prime} \rightarrow X^{\prime}$ over $K^{\prime}$. Here of course we write $X^{\prime}=X \otimes_{K} K^{\prime}$. By [3, Corollary 6.7.8], the $K^{\prime}$-scheme $\tilde{X}^{\prime}$ is geometrically normal. Since $\nu^{\prime}$ is birational, $\nu^{\prime}$ must be the normalization map of $X^{\prime}$, and remains so after any base field extension of $K^{\prime}$.

Since the field extension $K \subset K^{\prime}$ is finite and purely inseparable, there is an integer $n \geq 0$ with the property $\lambda^{p^{n}} \in K$ for all $\lambda \in K^{\prime}$. By the universal property of splitting fields, there exists a homomorphism $i: K^{\prime} \rightarrow K$ so that the composite $K \subset K^{\prime} \stackrel{i}{\hookrightarrow} K$ equals the $n$-fold Frobenius map. Consequently, $\tilde{X}^{\prime} \otimes_{K^{\prime}} K$ is the normalization of $X^{\left(p^{n}\right)}=X^{\prime} \otimes_{K^{\prime}} K$, where the tensor products are with respect to $i: K^{\prime} \rightarrow K$. This concludes the proof, since we saw in the preceding paragraph that $\tilde{X}^{\prime}$ is geometrically normal.

In this context, the following fact seems noteworthy as well.

Lemma 1.3. If $X^{(p)}$ is normal, then $X$ is geometrically normal.

Proof. According to Serre's Criterion, the condition that $X^{(p)}$ is normal means that it is regular in codimension $\leq 1$ and satisfies Serre's Condition $\left(S_{2}\right)$. Using that the canonical projection $X^{(p)} \rightarrow X$ is a homeomorphism, we infer with [2, Theorem 22.5.8] that $X$ must be geometrically regular in codimension $\leq 1$. Furthermore, [3, Corollary 6.7.8] implies that $X$ and all its base changes satisfy $\left(S_{2}\right)$. We conclude that $X$ is geometrically normal.

\section{Genus Change for algebraic curves}

Now let $X$ be a proper normal curve over $K$. Recall ([5, Chapter III, Section 7]) that the dualizing sheaf of $X$ exists and is invertible and is given by

$$
\omega_{X / K}=\mathcal{E} x t^{n-1}\left(\mathcal{O}_{X}, \mathcal{O}_{\mathbb{P}^{n}}(-n-1)\right)
$$

for any embedding $X \subset \mathbb{P}^{n}$. As in the preceding section, we also assume that $X$ is geometrically integral. The degree of an invertible sheaf $\mathcal{L}$ on $X$ is defined as the integer $\operatorname{deg}(\mathcal{L})=\chi(\mathcal{L})-\chi\left(\mathcal{O}_{X}\right)$. The main result of this paper relates the degrees of the dualizing sheaves on the Frobenius pullback and its normalization. We formulate it in terms of the relative dualizing sheaf:

Theorem 2.1. Let $\nu: Y \rightarrow X^{(p)}$ be the normalization map. Then the degree of the relative dualizing sheaf $\omega_{Y / X^{(p)}}$ is divisible by $p-1$.

Proof. The idea is to compute with relative dualizing sheaves on $X$. Since $X$ is normal, there is a unique morphism $f: X \rightarrow Y$ with $F_{X / K}=\nu \circ f$. The various relative dualizing sheaves satisfy

$$
\omega_{X / X^{(p)}}=\omega_{X / Y} \otimes f^{*}\left(\omega_{Y / X^{(p)}}\right) .
$$


Similarly we have $\omega_{X / K}=\omega_{X / X^{(p)}} \otimes F_{X / K}^{*}\left(\omega_{X^{(p)} / K}\right)$. Together with the formula $F_{X / K}^{*}\left(\omega_{X^{(p)} / K}\right)=F_{X}^{*}\left(\omega_{X / K}\right)=\omega_{X / K}^{\otimes p}$, this yields

$$
\omega_{X / X^{(p)}}=\omega_{X / K}^{\otimes(1-p)} .
$$

On the other hand, it follows from results of Kiehl and Kunz that the morphism $f: X \rightarrow Y$ locally admits $p$-bases ([6, Korollar 2 of Satz 5]). Therefore the sheaf of relative Kähler differentials $\Omega_{X / Y}^{1}$ is locally free of finite rank. It is related to the relative dualizing sheaf by

$$
\omega_{X / Y}=\operatorname{det}\left(\Omega_{X / Y}^{1}\right)^{\otimes(1-p)},
$$

according to loc. cit., Satz 9. Substituting formula (3) and (2) into (11), we infer that the degree of $f^{*}\left(\omega_{Y / X(p)}\right)$ is divisible by $p-1$. Finally, observe that $\operatorname{deg}\left(f^{*}\left(\omega_{Y / X^{(p)}}\right)\right)=\operatorname{deg}(f) \cdot \operatorname{deg}\left(\omega_{Y / X^{(p)}}\right)$, by the projection formula. Clearly, the surjection $f: X \rightarrow Y$ is purely inseparable; hence its degree is a $p$-power. From this we infer that the degree of $\omega_{Y / X^{(p)}}$ must be divisible by $p-1$.

Actually, the preceding result is equivalent to the following seemingly stronger statement.

Theorem 2.2. Let $K \subset L$ be an arbitrary field extension, and $Y$ be the normalization of $X \otimes_{K} L$. Then the degree of the relative dualizing sheaf $\omega_{Y / X \otimes_{K} L}$ is divisible by $p-1$.

Proof. First note the following transitivity property: Suppose $K \subset L^{\prime} \subset L$ is an intermediate field. Let $Y^{\prime}$ be the normalization of $X \otimes_{K} L^{\prime}$. Then $Y$ is the normalization of both $X \otimes_{K} L$ and $Y^{\prime} \otimes_{L^{\prime}} L$, and we have

$$
\omega_{Y / X \otimes_{K} L}=\omega_{Y / Y^{\prime} \otimes_{L^{\prime}} L} \otimes \varphi^{*}\left(\omega_{Y^{\prime} / X \otimes_{K} L^{\prime}} \otimes_{L^{\prime}} L\right),
$$

where $\varphi: Y \rightarrow Y^{\prime} \otimes_{L^{\prime}} L$ is the normalization map. Clearly, if two of the three dualizing sheaves have degree divisible by $p-1$, so has the third.

Using this transitivity property, we now settle the special case that the field $L$ in our field extension $K \subset L$ is perfect. Choose an integer $n \geq 0$ so that the normalization $Y^{\prime}$ of the Frobenius pullback $X^{\left(p^{n}\right)}$ is geometrically normal, as in Lemma 1.2. Since the field $L$ is perfect, there exists precisely one homomorphism $i: K \rightarrow L$ so that the composition $i \circ \mathrm{Fr}^{n}$ is our given extension $K \subset L$, according to [1, Chap. V, $\S 5$, No. 2, Proposition 3]. Consider the intermediate field $L^{\prime}=i(F)$. Induction on $n$, together with the transitivity property and Theorem 2.1, shows that the degree of $\omega_{Y^{\prime} / X \otimes_{K} L^{\prime}}$ is divisible by $p-1$. Since $Y^{\prime}$ is geometrically normal, we have $Y=Y^{\prime} \otimes_{L^{\prime}} L$. Another application of the transitivity property yields that the degree of $\omega_{Y / X \otimes_{K} L}$ is divisible by $p-1$, as desired.

It remains to treat the general case. Choose a perfect closure $L \subset L^{p^{-\infty}}$. According to the preceding paragraph, the theorem holds true for the field extensions $K \subset L^{p^{-\infty}}$ and $L \subset L^{p^{-\infty}}$. By transitivity, it must hold for $K \subset L$ as well.

We now may retrieve Tate's result.

Corollary 2.3 (Tate). Let $K \subset L$ be an arbitrary field extension, and let $Y$ be the normalization of $X \otimes_{K} L$. Then the difference $h^{1}\left(\mathcal{O}_{X}\right)-h^{1}\left(\mathcal{O}_{Y}\right)$ is divisible by $(p-1) / 2$. 
Proof. According to Lemma 1.1, the $L$-scheme $Y$ is geometrically integral. In particular, we have $K=H^{0}\left(X, \mathcal{O}_{X}\right)$ and $L=H^{0}\left(Y, \mathcal{O}_{Y}\right)$, whence

$$
h^{1}\left(\mathcal{O}_{X}\right)-h^{1}\left(\mathcal{O}_{Y}\right)=\chi\left(\mathcal{O}_{Y}\right)-\chi\left(\mathcal{O}_{X}\right)=\frac{1}{2}\left(\operatorname{deg}\left(\omega_{X / K}\right)-\operatorname{deg}\left(\omega_{Y / K}\right)\right),
$$

the latter by Serre duality and Riemann-Roch. The term on the right is nothing but $-\frac{1}{2} \operatorname{deg}\left(\omega_{Y / X \otimes_{K} L}\right)$, so the statement follows from Theorem 2.2.

\section{ACKNOWLEDGEMENT}

I wish to thank Igor Dolgachev for stimulating discussions.

\section{REFERENCES}

[1] N. Bourbaki, Algebra II. Chapters 4-7. Springer, Berlin, 1990. MR1080964 (91h:00003)

[2] A. Grothendieck, Éléments de géométrie algébrique IV: Étude locale des schémas et des morphismes de schémas I. Publ. Math., Inst. Hautes Études Sci. 20 (1964). MR0173675 (30:3885)

[3] A. Grothendieck, Éléments de géométrie algébrique IV: Étude locale des schémas et des morphismes de schémas II. Publ. Math., Inst. Hautes Études Sci. 24 (1965). MR0199181|(33:7330)

[4] A. Grothendieck, Éléments de géométrie algébrique IV: Étude locale des schémas et des morphismes de schémas III. Publ. Math., Inst. Hautes Études Sci. 28 (1966). MR0217086 (36:178)

[5] R. Hartshorne, Algebraic geometry. Grad. Texts in Math. 52. Springer-Verlag, Berlin, 1977. MR0463157 (57:3116)

[6] R. Kiehl, E. Kunz, Vollständige Durchschnitte und p-Basen. Arch. Math. 16 (1965), 348-362. MR0188220 (32:5659)

[7] N. Shepherd-Barron, Geography for surfaces of general type in positive characteristic. Invent. Math. 106 (1991), 263-274. MR1128215 (92k:14033)

[8] J. Tate, Genus change in inseparable extensions of function fields. Proc. Amer. Math. Soc. 3 (1952), 400-406. MR0047631 (13:905b)

Mathematisches Institut, Heinrich-Heine-Universität, 40225 Düsseldorf, Germany

E-mail address: schroeer@math.uni-duesseldorf.de 